\title{
Dynamic e-ICIC using Moving Average Crossover
}

\author{
Yuri V. L. de Melo, Vicente A. Sousa Jr. and Tarcisio F. Maciel
}

\begin{abstract}
Heterogeneous Networks (HetNets) are considered a technology option capable of improving system capacity and spatial spectrum reuse. However, high spectrum reuse causes high inter-cell interference among macro and small cells. Almost Blank Subframe (ABS) is a method of the Enhanced Inter-Cell Interference Coordination (e-ICIC) framework proposed for LTE systems as a means to mitigate interference among macro and small cells, which mutes some of macro cell transmissions in selected subframes to reduce interference to small cells, orthogonalizing macro and small cell transmissions in time-domain. In this work, we use Moving Average Crossover (MAC) based on trading know-how to propose a new dynamic ABS e-ICIC algorithm. Using system-level simulations, we attest that the proposed algorithm outperforms the best fixed e-ICIC by $6.4 \%$ in terms of capacity.
\end{abstract}

Index Terms-ABS, e-ICIC, Trend indicators, Trading know-how.

\section{INTRODUCTION}

According to the 3GPP [1], spatial spectrum reuse, extended coverage, and a combination of macro and small cells, namely a HetNet, can deal with the exponential increase in cellular capacity demand [2]. However, a significant level of inter-cell interference occurs not only due to the geographical proximity among cells but also because of the high transmission power of macro cells. Thus, the inter-cell interference increases as the spatial spectrum reuse gets higher, which negatively affects communication links. To manage the HetNet inter-cell interference, 3GPP introduced the ICIC in Release 8 [1], and the e-ICIC framework in Release 10 [3]. The e-ICIC framework incorporates the Almost Blank Subframe (ABS) method to coordinate, in time-domain, the subframes used for macro and small cell transmissions. Some ABS subframes are thus dedicated only to payload transmission of small cells, during which macro cells send only low-power control signals. Thus, users connected to small cells can experience lower inter-cell interference at the cost of macro cell's users performance. In particular, the ABS functionality can disable LTE transmissions by specifying an ABS pattern within a duty-cycle period of $40 \mathrm{~ms}$. This pattern is implemented with a bit-mask of 40 values (one per LTE subframe) [4].

The first ABS approaches, as in [5], were based on a fixed ABS ratio, i.e., a fixed percentage of ABS subframes was defined within a time window. Recent approaches dynamically change the ABS parameters in accordance with the network environment [6]. In this work, we associate trend indicators [7]

Yuri Melo and Tarcisio Maciel are with Wireless Telecommunications Research Group (GTEL), Teleinformatics Engineering Deparment, Federal University of Ceará (e-mail: \{yurivictor, maciel\}@gtel.ufc.br). Vicente Sousa is with Federal University of Rio Grande do Norte (e-mail: vicente.sousa@ufrn.edu.br). Tarcisio Maciel would like to acknowledge CNPq for its financial support under the grants 426385/2016-0, and 308621/2018-2. This study was financed in part by the Coordenação de Aperfeiçoamento de Pessoal de Nível Superior - Brasil (CAPES) - Finance Code 001. The proof of concept simulations provided by this paper was supported by High Performance Computing Center at UFRN (NPAD/UFRN).

Digital Object Identifier (DOI):10.14209/jcis.2019.9 and trading know-how [8] to dynamically monitor and modify ABS parameters as to improve the HetNet performance.

The remainder of this work is organized as follows. We present related works and highlight the contributions of our work in Section II. Before presenting the proposed dynamic e-ICIC algorithm in Section V, we introduced the system modeling in Section III, and the financial tools in Section IV. Section VI provides simulation results and discussions while final comments are addressed in Section VII.

\section{Literature Review and Main Contributions}

The authors of [5] propose a fixed ABS method with three ABS patterns: random, overlapping or merged. Although the ABS ratio remains fixed, small cells experience different interference profile according to the selected ABS pattern. In [9], the authors formulate the e-ICIC as an optimization problem. They propose to decompose the problem into three phases: (i) several single cell optimization problems; (ii) coordination among cells; and (iii) final User Equipment (UE) association. The solution is still based on a fixed ABS ratio, and the authors point out the need of efficient algorithms to estimate its desired value. The performance of multi-tier and dense deployed HetNets is assessed in [6], with fixed ABS e-ICIC applied to help the performance of edge users. The authors attested that a new design of interference mitigation solution in a $5^{\text {th }}$ Generation (5G) HetNet is essential.

Dynamic e-ICIC is addressed in some works [10]-[14]. Time and frequency domain ICIC techniques using reinforcement learning are devised in [10]. Dynamic e-ICIC substantially outperforms static e-ICIC approaches. The authors in [11] present a dynamic e-ICIC by adapting the ABS in response to variations in network load. They attested the performance gains along different indicators (such as spectral efficiency, fairness, mean file transfer times), assessing the system performance under different mobility scenarios, traffic mixes, and adaptation rate. In [12], standardized signaling is used to devise solutions for dynamic e-ICIC in LTE-A Rel-12 for both centralized and distributed structures. Simulations have shown stable performance gains for cell-edge throughput without loss in cell-average throughput. Performance evaluation based on site-specific data from a European capital is presented in [13]. A fast e-ICIC muting adaptation is proposed and compared to the semi-static e-ICIC. They concluded that the traditional semi-static e-ICIC leads to modest gains in realistic deployments, whereas the proposed fast dynamic e-ICIC leads to meaningful capacity gains. In [14], the algorithm checks the load at the macro/small cell layer and then decides whether the ABS shall be used. It achieved capacity gains in a Multiple Input Multiple Output (MIMO) scenario.

In this paper, we use some financial-based concepts to propose a new dynamic e-ICIC algorithm for HetNet. Previous works did not consider such approach, and we claim for the 
following contributions: (i) to adopt trading know-how to the dynamic e-ICIC; (ii) to propose a simple and efficient method that analyzes each subframe by trend indicators, avoiding the creation of ABS lists; (iii) to provide system-level analyses of the proposed algorithm in a HetNet scenario, following 3GPP's evaluation methodology; and (iv) to show the performance of the HetNet with and without ABS by means of simulations.

\section{System Model}

Our HetNet model consists of four hexagonal small cells of radius $r$ inside a macro cell coverage area. UEs are uniformly distributed over the cells' coverage areas, being 10 UEs in the macro cell area and 20 UEs in each small cell, totaling 90 UEs in the system. All UEs and Base Stations (BSs) are equipped with a single omnidirectional antenna.

Fig. 1 illustrates our HetNet deployment, identifying three important areas: macro cell, small cell's center, and small cell's edge. It also illustrates the temporal behavior of ABS (exclusive transmission to UEs at small cell's edge) and non-ABS (simultaneous transmission to UEs at macro cell and small cell's center areas) situations. The cell edge region comprises the area from a radius of $0.95 \cdot r$ up to $r$, inside the small cell hexagon, where the edge UEs are located, thus coping to approximately $10 \%$ of the small cells coverage area.

The macro and small cell pathloss models consider Line of Sight (LOS) and Non-Line of Sight (NLOS) propagation, which are modeled according to the urban macro (UMa) model detailed in [15], [16]. Our parameters are shown in Table I.

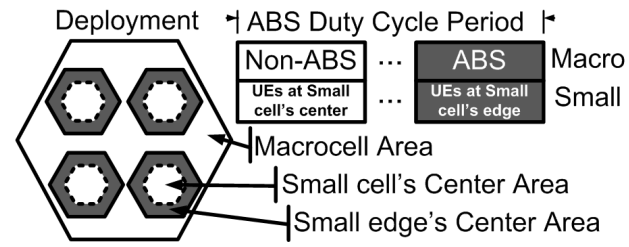

Fig. 1. System deployment and ABS modeling.

TABLE I

SYSTEM-LEVEL SIMULATOR PARAMETERS.

\begin{tabular}{lll}
\hline Parameter & Value & Source \\
\hline Frequency carrier & $2 \mathrm{GHz}$ & {$[17]$} \\
Bandwidth $(B)$ & $1.4 \mathrm{MHz}$ & {$[17]$} \\
UE speed & $3 \mathrm{~km} / \mathrm{h}$ & {$[18]$} \\
Cell radius (meters) & $1500(\mathrm{Macro})$ & {$[19]$} \\
Dist. from macro to small cells & $200(\mathrm{Small})$ & \\
Number of small cells $(T)$ & $400 \mathrm{~m}$ & {$[16]$} \\
Macro transmit power $\left(p_{m}\right)$ & $46 \mathrm{dBm}$ & {$[17]$} \\
Small transmit power $\left(p_{s}\right)$ & $36 \mathrm{dBm}$ & {$[17]$} \\
Macro/Small pathloss $(\mathrm{LOS})$ & $-22 \log _{10}(d)-34.02 \mathrm{~dB}$ & {$[15],[16]$} \\
Macro pathloss (NLOS) & $-39.01 \log _{10}(d)-21.56 \mathrm{~dB}$ & {$[15],[16]$} \\
Small pathloss (NLOS) & $-36.9 \log _{10}(d)-30.5 \mathrm{~dB}$ & {$[15],[16]$} \\
Shadowing std. dev. & $8 \mathrm{~dB}(\mathrm{Macro})$ & {$[20]$} \\
Small scale fading model & $10 \mathrm{~dB}(\operatorname{Small})$ & {$[21]$} \\
Thermal noise power $(\eta)$ & Clarke/Gans & {$[20]$} \\
Scheduling policy & $-112 \mathrm{dBm}$ & {$[22]$} \\
\hline is the distance between the transmitter and the receiver in meters.
\end{tabular}

Our performance evaluation is based on Downlink (DL) capacity computed by Shannon-Hartley formulation as $C_{u, k}^{\text {macro }}=B \log _{2}\left(1+\gamma_{u, k}^{\text {macro }}\right)$, where $B$ is the channel bandwidth, $u$ is a UE in the macro cell with $u \in\{1,2, \ldots, U\}$, $k$ is the subframe index, and $\gamma_{u, k}^{\text {macro }}$ is the macro cell Signal to Interference-plus-Noise Ratio (SINR) calculated as:

$$
\gamma_{u, k}^{\text {macro }}=\frac{\left|h_{u, k}\right|^{2} p_{m}}{\sum_{t}^{T}\left|h_{u, k}^{(t)}\right|^{2} p_{s}+\eta},
$$

with $h_{u, k}$ being the channel coefficient (comprising pathloss, shadowing, and small scale fading); $t, t^{\prime} \in\{1,2, \ldots, T\}$ are indices for small cells; and $\eta$ is the Additive White Gaussian Noise (AWGN) noise power. Thus, the macro cell mean capacity is computed as $C_{\text {mean }}^{\text {macro }}=\frac{1}{N_{s f}} \sum_{k=1}^{N_{s f}} \sum_{u=1}^{U} C_{u, k}^{\text {macro }}$, where $N_{s f}$ is the total number of subframes.

In order to calculate the mean capacity for small cells, we define four scenarios: (i) Homogeneous (20 UEs uniformly distributed at each small cell area); (ii) Heterogeneous (disjoint sets of 10 UEs uniformly distributed at center and edge areas of small cell); (iii) Heterogeneous with fixed ABS e-ICIC (based on 3GPP Rel. 10); and (iv) Heterogeneous with dynamic ABS e-ICIC (our proposed solution).

As specified in 3GPP Rel. 10, fixed ABS e-ICIC approach defines an ABS ratio to control interference. For instance, an ABS ratio of $30 \%$ means that the macro cell gates off $30 \%$ of duty-cycle time $(40 \mathrm{~ms})$ and transmits in the rest (simultaneously with small cell's center area). There is no ABS for the first two scenarios (Homogeneous and Heterogeneous), thus the SINR of a UE $j$ connected to a small cell $t$, in subframe $k$ is $\gamma_{j, k}^{(t), \text { small }}=\frac{\left|h_{j, k}^{(t)}\right|^{2} p_{s}}{\left|h_{j, k}\right|^{2} p_{m}+\sum_{t^{\prime} \neq t}^{T}\left|h_{j, k}^{\left(t^{\prime}\right)}\right|^{2} p_{s}+\eta}$.

Then, for a small cell $t$, the capacity is $C_{j, k}^{(t), \text { small }}=$ $B \log _{2}\left(1+\gamma_{j, k}^{(t), \text { small }}\right)$, and the mean capacity of the small cells is computed as $C_{\text {mean }}^{\text {small }}=\frac{1}{N_{s f} \cdot T} \sum_{t=1}^{T} \sum_{k=1}^{N_{s f}} \sum_{j=1}^{J} C_{j, k}^{(t), \text { small }}$.

For scenarios with $\mathrm{ABS}$, the macro cell decides which subframes will be used or not (and sends the ABS pattern to small cells). The macro cell mean capacity during ABS subframes is equal to zero, because there is no macro cell payload transmission. On the other hand, the SINR of macro cell UEs during non-ABS is computed as in Eq. (1) with interference being from the UEs at the small cell's center area.

As for the heterogeneous scenario perspective when ABS e-ICIC is employed, UEs of small cells are split into two sets: (i) UEs located at small cell's center area, $m \in\{1,2, \ldots, M\}$, and; (ii) UEs located at small cell's edge area, $l \in\{1,2, \ldots, L\}$. Thus, the SINR of user $l$ connected to small cell $t$ in subframe $k$ during ABS is $\gamma_{l, k}^{(t) \text {,small }}=\frac{\left|h_{l, k}^{(t)}\right|^{2} p_{s}}{\sum_{t^{\prime} \neq t}^{T}\left|h_{l, k}^{\left(t^{\prime}\right)}\right|^{2} p_{s}+\eta}$ and the corresponding capacity is $C_{l, k}^{(t) \text {,small }}=B \log _{2}\left(1+\gamma_{l, k}^{(t) \text {,small }}\right)$.

For non-ABS (UEs located at the center area experiencing macro cell interference), we could rewrite the SINR as $\gamma_{m, k}^{(t), \text { small }}=\frac{\left|h_{m, k}^{(t)}\right|^{2} p_{s}}{\left|h_{m, k}\right|^{2} p_{m}+\sum_{t^{\prime} \neq t}^{T}\left|h_{m, k}^{\left(t^{\prime}\right)}\right|^{2} p_{s}+\eta}$, and the corresponding capacity as $C_{m, k}^{(t) \text {,small }}=B \log _{2}\left(1+\gamma_{m, k}^{(t) \text {,small }}\right)$. Finally, the mean capacity of small cell regarding $\mathrm{ABS}$ and non-ABS situations is 


$$
C_{\text {mean }}^{\text {small }}=\frac{1}{N_{s f} \cdot T} \sum_{t=1}^{T} \sum_{k=1}^{N_{s f}}\left(\sum_{m=1}^{M} C_{m, k}^{(t), \text { small }}+\sum_{l=1}^{L} C_{l, k}^{(t), \text { small }}\right) .
$$

IV. Financial tools

Trend indicators are mathematical tools applied in several fields of knowledge, such as engineering, finance and biology. Their main purpose is to determine the prevailing trend of a target data within a reference range. Among several trend indicators, the Moving Average (MA) is considered the most widespread and practical in data analysis [7], [8]. This is the main reason of using MA as trend measure of our solution.

We can define MA as a General Weighted Moving Average at time $t$ in the time-series $\{X\}$ by $M A_{t}(n)=\frac{\sum_{i=\alpha}^{\beta} w_{i} X_{t-i}}{\sum_{i=\alpha}^{\beta} w_{i}}$, where $n=\beta-\alpha+1$ is a fixed size window, also called the averaging period, over which the MA is computed, and $w_{i}$ is a weight that characterizes the importance of one data against another [23]. The moving effect happens because, while the newest data sample is injected, the oldest is ejected from the MA.

Different configurations of parameters define different MA measures. An MA suitable for forecasting is the Right Aligned Moving Average $\left(\frac{1}{n} \sum_{i=0}^{n-1} X_{t-i}\right)$, also with $w_{i}=1, \alpha=0$, and $\beta=n-1$. Only the current and past data are inputs for this MA. It is also named as Simple Moving Average (SMA). We can also define the Exponential Moving Average (EMA) by weighting data with a decay factor $w_{i}=\lambda^{i}$. By setting the value of $\lambda(0<\lambda<1)$, we can give greater or lesser weight to the most recent data. Comparing SMA to EMA, the SMA is less reactive to data changes while the EMA is more reactive, i.e., EMA responds faster to new data than the SMA.

Two important characteristics of all MAs are that they smooth and lag the input data. Their use and application is almost always a trade-off between these characteristics. The smoothing function eliminates higher frequency components of the input data, so MA are also referred to as low pass filters. When it comes to the lag time, all moving averages are called lagging indicators, because they always happen after the fact. Thus, managing the lag is essential to control the speed of trend-following behavior of MA. As introduced in [23], the average lag time $v$ of a weighted moving average is computed as $v(M A(n))=\frac{\sum_{i=1}^{n-1} w_{i} \cdot i}{\sum_{i=0}^{n-1} w_{i}}$. Then, the average lag time of SMA is $v(S M A(n))=\frac{n-1}{2}$, and of EMA is $v(E M A(\lambda, n))=\frac{\lambda-\lambda^{n}}{(1-\lambda)\left(1-\lambda^{n}\right)}-\frac{(n-1) \lambda^{n}}{1-\lambda^{n}}$.

We need those equations to control the lag time of the proposed Moving Average Crossover (MAC) ABS e-ICIC.

A trend-following strategy is based on switching between the market and the cash depending on whether the market prices trend upward or downward [23]. It provides two types of signals to help the switching decision: Buy and Sell signals. A Buy signal is an indication to invest in the stocks, whereas a Sell signal is an indication to sell the stocks and invest in cash [23]. When the trend strategy identifies that prices trend upward (downward), it generates a Buy (Sell) trading signal.

The technical trading based on MAC was proposed in 1935 by Gartley [24]. The idea of MAC is to generate a trading signal from two MAs with different window sizes: $M A C_{t}\left(n_{1}, n_{2}\right)=M A_{t}\left(n_{1}\right)-M A_{t}\left(n_{2}\right)$, with $n_{2}>n_{1}$. A crossover occurs when the shorter MA (i.e. $\left.M A_{t}\left(n_{1}\right)\right)$ crosses either above or below the longer MA (i.e. $\left.M A_{t}\left(n_{2}\right)\right)$. Then, Buy and Sell signals are generated as follows:

- Buy signal (Bullish crossover/Golden cross):

- $M A C_{t-1}\left(n_{1}, n_{2}\right)<0$ and $\operatorname{MAC}_{t}\left(n_{1}, n_{2}\right)>0$;

- Sell signal (Bearish crossover/Death cross):

- $M A C_{t-1}\left(n_{1}, n_{2}\right)>0$ and $M A C_{t}\left(n_{1}, n_{2}\right)<0$.

In a filter perspective, as discussed in [25], the higher the number of elements in MA sum (window size), the lower is its cutoff frequency. As the MAC is a combination of two lowpass filters with distinct cutoff frequencies (MAs with different window sizes), the MAC signal refers to a bandpass filter output. The investigation of the MAC bandwidth is out of the scope of this proposal but addressed to further studies. In the next section, we propose how to adapt the MAC to the ABS e-ICIC problem.

\section{Algorithm proposal: MAC for the ABS}

Using financial-specific nomenclature, our ABS e-ICIC based on MAC tool is defined as:

- we define the time (independent variable) as subframe $k$ instead of minutes, hours or years and, consequently, the window size $n$ is measured in number of subframes;

- the price, which is used to provide crossover in trading, is replaced by macro cell capacity;

- we define the trading operation interval as 40 subframes;

- to increase the crossover probability during the trading operation interval, we define our MAC signal based on SMA and EMA, with the same lag time, as $M A C_{k}(n)=E M A_{k}(n)-S M A_{k}(n)$.

The modified EMA, to have the same lag time of the SMA, is $\operatorname{EMA}(n)=(1-\lambda) \sum_{i=0}^{\infty} \lambda^{i} X_{t-i}$, where $\lambda=\frac{n-1}{n+1}$.

Algorithm 1 shows the pseudo-code of the proposed MAC e-ICIC algorithm. Initially, we define all subframes as non-ABS as well as some input parameters (e.g., window size $n$ and threshold $\varepsilon$, the latter responsible for allowing or not subframes to be set as ABS). Inside the loop occur not only the calculation of SINR and capacity but also the test whether the MA window is complete or not. If true, $M A C_{k}$ and $M A C_{k-1}$ are calculated. After that, if the current capacity $C_{u, k}^{\text {macro }}$ is below the threshold $\varepsilon$, and there is a Sell signal, the next subframes are defined as ABS until a Buy signal happens.

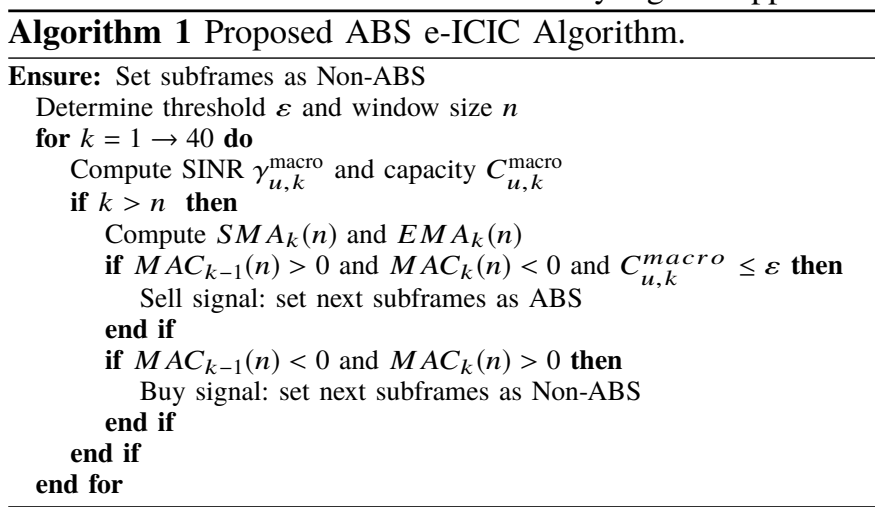




\section{Results}

Fig. 2 shows the mean capacity of macro and small cells for the first three scenarios defined in the Section III. Plots from macro and small cells are overlapped so that the total mean capacity can be visualized. As expected, due to the concentration of UEs in the small cell's edge area, the total mean capacity (i.e., macro cell plus small cells) in the Heterogeneous scenario is smaller than that in the Homogeneous. Although the macro cell capacity is the same, a loss of $20 \%$ is experienced in the Heterogeneous scenario compared to the Homogeneous one. Considering the Heterogeneous scenario with fixed ABS e-ICIC, the macro cell capacity decreases when the ABS ratio increases (more ABS subframes), while small cell capacity increases. Plots consider a $95 \%$ confidence interval.

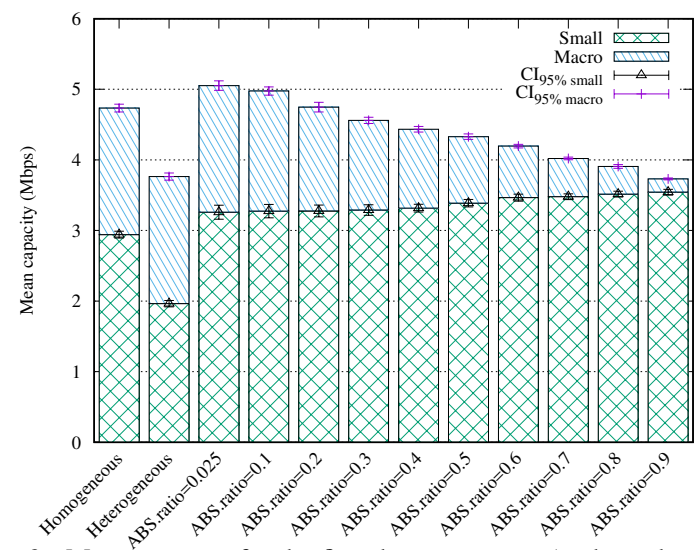

Fig. 2. Mean capacity for the first three scenarios (without dynamic e-ICIC).

Before showing results for the Heterogeneous scenario with dynamic ABS e-ICIC, we perform an investigative study about the algorithm's parameters (the $\varepsilon$ threshold and the window size $n$ ). To control the macro cell capacity loss due to excessive ABS subframes, the $\varepsilon$ threshold was set based on values from the 1 st to the 5 th percentile of macro cell capacity in the Homogeneous scenario $(60.2 \mathrm{kbps}$ and $218.5 \mathrm{kbps}$, respectively). On the other hand, we want to understand the influence of the window size into the amount of Buy and Sell signals inside a trading operation interval (40 subframes). Thus, we plot the macro cell mean capacity considering the percentile $p \in[0.01,0.05]$ and $n \in[5,30]$ in Fig. 3 .

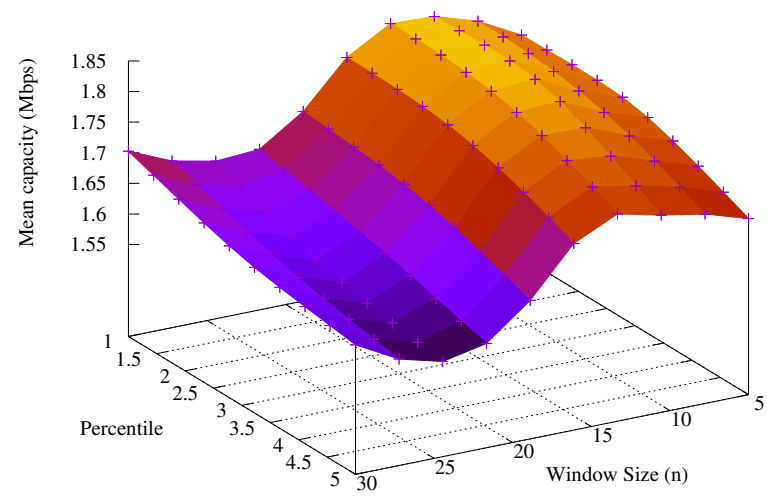

Fig. 3. Macrocell mean capacity (variation of $\varepsilon$ threshold, and window size).
TABLE II

BEARISH AND BULLISH CROSSOVERS FOR $p=3 \%$.

\begin{tabular}{|c|c|c|c|c|c|c|}
\hline Window size $(n)$ & 5 & 10 & 15 & 20 & 25 & 30 \\
\hline Bearish crossover (\%) & 51 & 52 & 53 & 88 & 100 & $100 \%$ \\
\hline Bullish crossover (\%) & 49 & 48 & 47 & 12 & 0 & 0 \\
\hline
\end{tabular}

Therein, the higher the $\varepsilon$ threshold, the lower the macro cell mean capacity, attesting the occurrence of more ABS subframes. When it comes to window size, the macro cell capacity is high for the region $n \in[5,15]$ (lack of false signals, and a good balance between Bearish and Bullish crossovers), whereas there is a macro cell capacity loss in the region $n \in[15,30]$ due to the lag time, and the low occurrence of Bullish crossovers. We attest those reasons in Table II, showing the percentage of bearish and bullish crossovers regarding the tested window sizes. Finally, we show the performance results for the Heterogeneous scenario with the proposed dynamic ABS e-ICIC based on MAC in Fig. 4. These results are identified in the figure with the MAC e-ICIC prefix, with specific window size and percentile value (which define the $\varepsilon$ threshold). Following the knowledge from Fig. 3 and Table II, we display results for window sizes of 10 and 15, and for the 1st and the 3rd percentiles. As expected, the macro cell capacity is still the same for all scenarios, attesting no significant loss due to the ABS. However, the relative capacity gains of our algorithm proposal are 14\% and 6.4\% compared to Homogeneous and Heterogeneous scenarios, respectively. Regarding the ABS ratio, the mean ABS ratio achieved by the proposed algorithm was 0.1145 , which is a ratio impossible to be set for the heterogeneous with fixed ABS e-ICIC case.

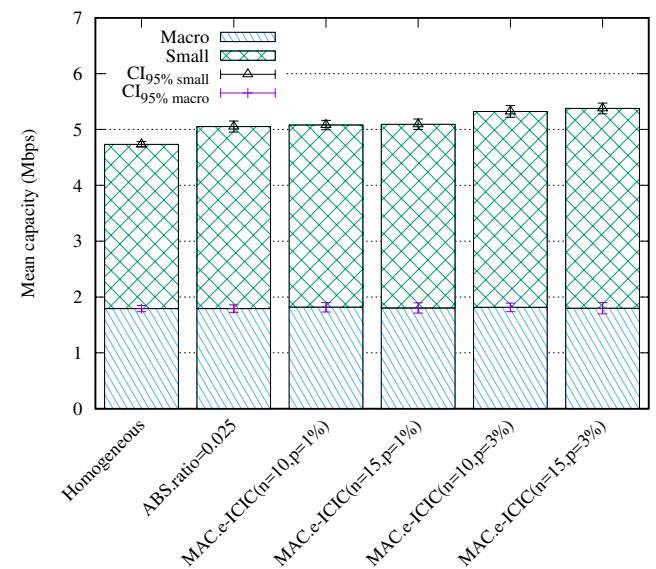

Fig. 4. Mean capacity of the proposed dynamic ABS e-ICIC based on MAC.

\section{Conclusions}

In this work, we propose a new ABS e-ICIC based on Moving Average Crossover to manage the interference in HetNets dynamically. The proposed algorithm was prototyped using an in-house built system-level simulator, following the recommendation of 3GPP standardization body. Results demonstrate gains of $6.4 \%$ and $14 \%$ compared to Heterogeneous and Homogeneous scenarios, respectively. Our next steps will extend our solution with other trend indicators, such as Bollinger Bands (BB), Average Directional Index (ADX), and Stop And Reverse (SAR) parabolic. 


\section{REFERENCES}

[1] J. Wannstrom and K. Mallinson, "Hetnet/small cells," http://www.3gpp. org/hetnet.

[2] 3GPP, "Vocabulary for 3GPP Specifications," 3rd Generation Partnership Project (3GPP), TR 21.905, 2018. [Online]. Available: http://www. 3gpp.org/ftp/Specs/html-info/21905.htm

[3] 3GPP, "Evolved universal terrestrial radio access (E-UTRA) and evolved universal terrestrial radio access network (E-UTRAN); overall description; stage 2," Technical Specification Group Radio Access Network, Evolved Universal Terrestrial Radio Access (E-UTRA), Tech. Rep., 2015.

[4] 3GPP, "TISPAN; PSTN/ISDN simulation services; Extensible Markup Language (XML) Configuration Access Protocol (XCAP) over the Ut interface for Manipulating NGN PSTN/ISDN Simulation Services," 3rd Generation Partnership Project (3GPP), TS 24.423, Mar. 2012. [Online]. Available: http://www.3gpp.org/ftp/Specs/html-info/24423.htm

[5] R. Singh and C. S. R. Murthy, "Techniques for interference mitigation using cooperative resource partitioning in multitier LTE HetNets," IEEE Systems Journal, vol. 12, no. 1, pp. 843-853, mar 2018, doi:10.1109/jsyst.2016.2527504.

[6] C. Ma, M. Ding, D. Lopez-Perez, Z. Lin, J. Li, and G. Mao, "Performance analysis of the idle mode capability in a dense heterogeneous cellular network," IEEE Transactions on Communications, vol. 66, no. 9, pp. 3959-3973, sep 2018, doi:10.1109/tcomm.2018.2822805.

[7] D. Guppy, Market Trading Tactics. John Wiley \& Sons, 2011.

[8] P. Glabadanidis, Market Timing and Moving Averages: An Empirical Analysis of Performance in asset allocation. Palgrave Macmillan US, 2015, doi:10.1057/9781137359834.

[9] J. Huang, J. Li, Z. Chen, and H. Pan, "HICIC: Hybrid inter-cell interference coordination for two-tier heterogeneous networks with non-uniform topologies," IEEE Access, vol. 6, pp. 34 707-34 723, 2018, doi:10.1109/access.2018.2841194.

[10] M. Simsek, M. Bennis, and A. Czylwik, "Dynamic inter-cell interference coordination in hetnets: A reinforcement learning approach," in 2012 IEEE Global Communications Conference (GLOBECOM), Dec 2012, pp. 5446-5450, doi:10.1109/GLOCOM.2012.6503987.

[11] S. Vasudevan, R. N. Pupala, and K. Sivanesan, "Dynamic e-ICIC - a proactive strategy for improving spectral efficiencies of heterogeneous LTE cellular networks by leveraging user mobility and traffic dynamics," IEEE Transactions on Wireless Communications, vol. 12, no. 10, pp. 4956-4969, October 2013, doi:10.1109/TWC.2013.081413.121651.

[12] Z. Xiong, M. Zhang, M. Baker, and H. Sun, "Dynamic ICIC in LTE-advanced networks for inter-cell interference mitigation," in 2015 IEEE 82nd Vehicular Technology Conference (VTC2015-Fall), Sept 2015, pp. 1-5, doi:10.1109/VTCFall.2015.7390903.

[13] K. I. Pedersen, B. Soret, S. Barcos, G. Pocovi, and H. Wang, "Study of dynamic eicic in a realistic urban deployment," in 2015 IEEE 81st Vehicular Technology Conference (VTC Spring), May 2015, pp. 1-5, doi:10.1109/VTCSpring.2015.7146112.

[14] B. Soret and K. I. Pedersen, "Centralized and distributed solutions for fast muting adaptation in lte-advanced hetnets," IEEE Transactions on Vehicular Technology, vol. 64, no. 1, pp. 147-158, Jan 2015, doi:10.1109/TVT.2014.2320056.

[15] 3GPP, "Further advancements for E-UTRA physical layer aspects," Third Generation Partnership Project, TR 36.814 V9.0.0, Mar. 2009.

[16] H. Claussen, D. Lopez-Perez, L. Ho, R. Razavi, and S. Kucera, Small Cell Networks: Deployment, Management, and Optimization. JOHN WILEY \& SONS INC, 2016.

[17] 3GPP, "FDD repeater radio transmission and reception," Technical Specification Group Radio Access Networks, TS 36.106 V15.0.0, 2018.

[18] 3GPP, "Coordinated multi-point operation for LTE physical layer aspects," Technical Specification Group Services and System Aspects, TS 36.819 V11.2.0, 2013.

[19] 3GPP, "Radio network planning aspects," Third Generation Partnership Project, TR 43.030 V15.0.0, 2018.

[20] 3GPP, "Physical layer aspects for evolved universal terrestrial radio access (UTRA)," Third Generation Partnership Project, TR 25.814 V7.1.0, 2006.

[21] Y. S. Cho, J. Kim, and W. Y. Yang, MIMO-OFDM Wireless Communications with MATLAB. John Wiley \& Sons, 2010.

[22] 3GPP, "Radio frequency (RF) system scenarios," Technical Specification Group Services and System Aspects, TS 36.942 V15.0.0, 2018.

[23] V. Zakamulin, Market Timing with Moving Averages: The Anatomy and Performance of Trading Rules. Springer International Publishing, 2017, doi:10.1007/978-3-319-60970-6.
[24] H. M. Gartley, Profits in the Stock Market/With Charts. Lambert Gann Publishing, 1935.

[25] P. Diniz, E. da Silva, and S. Netto, Digital Signal Processing: System Analysis and Design. Cambridge University Press, 2006, doi:10.1109/TVT.2014.2320056.

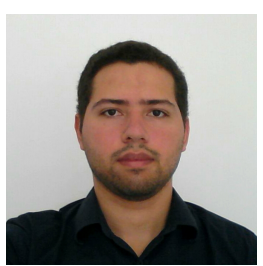

Yuri Victor Lima de Melo received the B.Sc. degree in Electrical Engineering from the Federal University of Rio Grande do Norte (UFRN), Natal, Brazil, in 2012. M.Sc. degree in Teleinformatics Engineering from the Federal University of Ceará (UFC), Fortaleza, Brazil, in 2015. Since 2016, he has been with the Federal University of Ceará, Brazil, where he is currently an Assistant Professor. His research interest include Device-to-Device (D2D) communications, power control, evolutionary computation and multi-access networks.

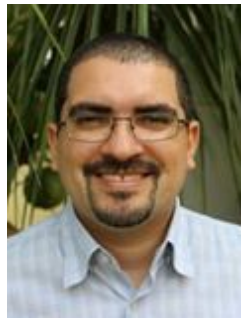

Vicente A. Sousa Jr received his B.Sc., M.Sc., and $\mathrm{Ph} . \mathrm{D}$. degrees in electrical engineering from Federal University of Ceará (UFC) in 2001, 2002, and 2009, respectively. Between 2001 and 2006, he developed solutions to UMTS/WLAN interworking for UFC and Ericsson of Brazil. Between 2006 and 2010, he contributed to WIMAX standardization and Nokia's product as a researcher at Nokia Technology Institute (INdT). He is now a professor at Federal University of Rio Grande do Norte (UFRN), Brazil.

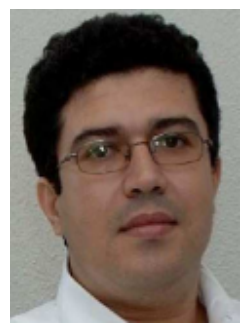

Tarcisio F. Maciel received his B.Sc., M.Sc., and Dr.-Ing. degrees in Electrical Engineering from the Federal University of Ceará (UFC) in 2002, from UFC in 2004, and from Technische Universität Darmstadt (TUD), Germany, in 2008, respectively. Since 2001 , he has actively participated in several cooperation projects between Wireless Telecommunications Research Group (GTEL), UFC, and Ericsson Research. From 2005 to 2008, he was a research assistant with the Communications Engineering Laboratory, TUD. Since 2008, he has been a member of the Post-Graduation Program in Teleinformatics Engineering, UFC. In 2009, he was a Professor of computer engineering with UFC-Sobral. From 2010 to 2015, he was a Professor with the Center of Technology, UFC. Since 2015, he is a Professor with the Teleinformatics Engineering Department, UFC. His research interests include wireless communications, signal processing, and optimization. 